\title{
A Multipurpose Composition Based on Brilliant Blue R for Developing Latent Fingerprints on Crime Scene Evidence
}

\begin{abstract}
Keywords: Fingerprints; Forensic; Small particle reagent
Abstract

The simplest and most commonly used method for detecting latent fingerprints is powder dusting. This technique relies on the mechanical adherence of the detecting composition to the moisture content of sweat. Under the present work, a novel, fluorescent, nontoxic and cost-effective powder composition for detection of latent fingerprints has been developed. The fluorescent component of the formulation is the brilliant blue R stain. The powder, when suspended in water, along with a few drops of a commercial liquid detergent acts as a small particle reagent (SPR) and develops latent fingerprints on non-porous wet surfaces. The composition has multipurpose utility since in powdered state; it develops sharp and detailed fingerprints on an array of articles removed from conventional crime scenes, while in SPR state lifts clear fingerprints on moist non-porous items.
\end{abstract}

\section{Introduction}

Latent fingerprints are valuable pieces of evidence that are often found when a crime has been committed. This type of evidence is hardly visible and therefore it is pertinent to institute a physical or chemical technique to visualize it. The powder technique for detecting latent fingerprints involves the application of a finely divided formulation to the finger mark impression, generally with a glass fiber or a camel hair brush. The powder is mechanically adhered to the sweat residue defining the ridge pattern of the fingertips. The furrows which contain extremely meager amount of sweat do not adsorb the powder on to them. The final outcome is that the powder formulation sticks to the ridges, but is easily blown off the furrows. Since the powder is normally colored, the ridge pattern becomes visible and the latent print is said to have developed.

Small particle reagent is considered to be a wet powdering method for development of latent fingerprints on broad spectrum crime scene evidence. It is sensitive to the lipid constituents of fingerprint sweat and is effective for processing fingerprints on articles that have been accidently or deliberately wetted [1]. In conventional small particle reagent, a suspension of molybdenum(IV) sulfide in a surfactant is used as a base material [2]. The crystal dimensions of molybdenum(IV) sulfide are critical for fingerprint development. The reason being that the quantum of attraction between molybdenum(IV) sulfide and the lipid deposition is influenced by the unit cell parameters of the former. However, as the base material is gray in color, the fingerprints developed on dark colored surfaces are not sufficiently clear due to lack of contrast. A formulation based on white basic zinc carbonate, the unit cell dimensions of which are comparable to those of molybdenum(IV) sulfide, serves to overcome this problem by developing sharp fingerprints on dark colored articles [3]. Titanium

\section{Journal of}

Forensic Investigation

\author{
G.S. Sodhi' ${ }^{*}$, Smily Kapoor ${ }^{2}$, Sanjiv Kumar ${ }^{3}$

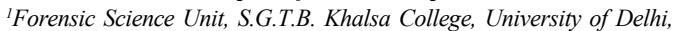 \\ India \\ ${ }^{2}$ University Science Instrumentation Center, University of Delhi, India \\ ${ }^{3}$ Department of Chemistry, Indira Gandhi National Open University, \\ Maidan Garhi, India

\section{Address for Correspondence} \\ Dr. G.S.Sodhi, Associate Professor, Department of Chemistry, S.G.T.B. \\ Khalsa college, University of Delhi, India; E-mail: sodhigs1@rediffmail.com \\ Submission: 09 May, 2014 \\ Accepted: 04 July, 2014 \\ Published: 06 July, 2014 \\ Copyright: $\odot 2014$ Kumar S, et al. This is an open access article \\ distributed under the Creative Commons Attribution License, which \\ permits unrestricted use, distribution, and reproduction in any medium, \\ provided the original work is properly cited.
}

dioxide may also be used for preparing a small particle reagent formulation. It develops white colored fingerprints on plastic, glass and metallic articles [4].

In this communication, we report the utility of a fluorescent fingerprint dusting composition based on brilliant blue $\mathrm{R}$ dye. It detects latent impressions on a host of porous and non-porous items. When suspended in water containing a few drops of a liquid detergent, the composition acts as small particle reagent and lifts fingerprints on wet non-porous articles. In many countries, including India, the first responders are the police personnel, most of who are from non-science background. A single composition which can develop latent prints on both wet and dry surfaces is deemed to be of great help to them.

\section{Experimental}

Basic zinc carbonate was purchased from Glaxo Laboratories, New Delhi, while brilliant blue R dye, titanium dioxide, lycopodium, gum rosin and zinc stearate were procured from Sigma-Aldrich India, New Delhi and used without further purification. Students and faculty members of our group with variable donor capabilities were asked to impinge latent fingerprints without prior washing and cleaning of hands. This ensured practical variability in the print samples. The fingers were first gently rubbed on the forehead and then pressed on the relevant surface. The entire project was conducted in winter season when the temperature was $18-20^{\circ} \mathrm{C}$ and relative humidity $30-75 \%$.

The powder formulation was prepared as follows: To a suspension of basic zinc carbonate $(3.0 \mathrm{~g})$ in $10 \mathrm{~mL}$ distilled water, a solution of brilliant blue $\mathrm{R}$ dye $(80 \mathrm{mg})$ in $5 \mathrm{ml}$ ethanol was added. To the mixture, titanium dioxide $(2.0 \mathrm{~g})$, lycopodium $(0.1 \mathrm{~g})$, gum rosin $(0.1$ $\mathrm{g})$, and zinc stearate $(0.1 \mathrm{~g})$ were successively added. The contents were thoroughly mixed, stirred and then allowed to dry under natural conditions. The solid mass was ground to a fine powder, the particle diameter being approximately $1 \mu \mathrm{m}$. The dye content in the composition was $1.5 \%$ by mass. 
The small particle reagent formulation was prepared by suspending $5.0 \mathrm{~g}$ of the powder in $75 \mathrm{~mL}$ water. A commercial liquid detergent, Genteel ${ }^{\mathrm{R}}(0.3 \mathrm{ml})$, the main ingredient of which is linear alkylbenzene sulfonate was added to the suspension and the contents were thoroughly shaken.

Surfaces considered for powder form. Latent fingerprints were developed on plastic sheet (of PET variety), wooden ply and glossy magazine paper. The composition was dusted on different surfaces containing the latent fingerprints with a camel hair brush. Thereafter, excess powder was blown off. The ridge pattern of the fingerprints was rendered visible. The fluorescence of the prints was observed in cyan polylight (Rofin; Model PRY-002) of wavelength $505 \mathrm{~nm}$.

Surfaces considered for SPR suspension form. Latent fingerprints were developed on metal surface (stainless steel knife) and the sticky (adhesive) side of vinyl-coated electrical tape, after these were immersed in clean water for 1 to 30 days. Fingerprints were also developed on these items, after these were immersed in dirty water for 1 to 30 days. The dirty water was collected from a drain which flows past our institute. About $200 \mathrm{~mL}$ lot of clean water and dirty water was placed in separate beakers. A set of three surfaces, bearing the latent impressions, were immersed in each beakers for 1 to 30 days. Thereafter, the test suspension was applied by immersing the substrate in the reagent for 30 to $60 \mathrm{sec}$ followed by washing in water and then dried with a hair dryer for 30 seconds. The developed fingerprints may be enhanced by illumination with radiation of 505 $\mathrm{nm}$ wavelength.

The developed prints were captured by digital camera (GE X5) in the auto mode. All the photographs were taken with full resolution (14 mega pixels) to capture finer details. The images were stored in the jpeg format for record.

\section{Results and Discussion}

The fluorescent characteristic of the composition, both in the powder and SPR state, assists in enhancing weak fingerprints that are frequently encountered at scenes of crime. The effectiveness with which the powder adheres to the ridges depends on the size and shape of the particles that compose the formulation. Small, fine particles, having diameter around $1 \mu \mathrm{m}$, adhere more easily than larger ones [5]. Good quality prints have been lifted from plastic sheet, wooden ply and glossy paper. A sample fingerprint developed on plastic sheet (PET), wooden ply and magazine paper respectively using dry powder formulation is shown in Figure 1. Each print was developed after a gap of 4 days. The quality of developed prints is not compromised even after a lapse of 90 days.

Small particle reagent technique has proved its worth in detecting fingerprints on moist, smooth surfaces. The novel composition developed sufficiently clear and identifiable fingerprints on metallic surface (knife) samples which had remained in water for up to 20 days. Thereafter, the quality of developed prints decreases. A sample fingerprint developed respectively on metallic surface and plastic tape after 5 days of immersion in clean water is shown in Figure 2. On metallic surface, clear and sharp prints could be developed until 18 to 20 days after immersion into clean water, while on electrical tape; sharp prints could be developed after 15 days of immersion into clean water. Thus, better results were obtained on metallic surface as

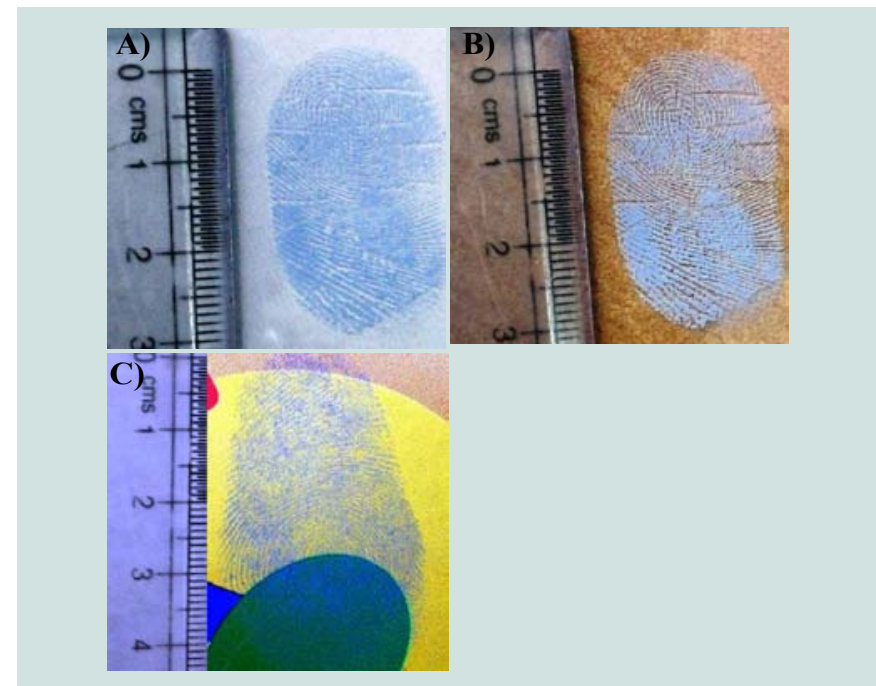

Figure 1: Fingerprints developed on (A) plastic sheet; (B) wooden ply; (C) magazine paper using dry powder formulation.

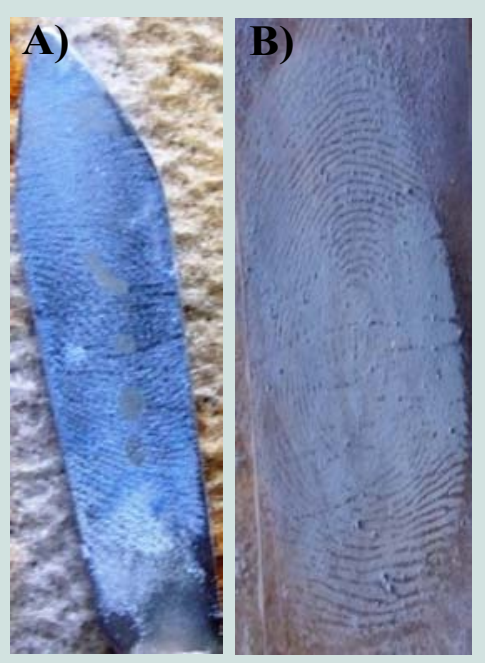

Figure 2: Fingerprints developed on (A) metallic surface; (B) plastic tape; after 5 days of immersion in clean water.

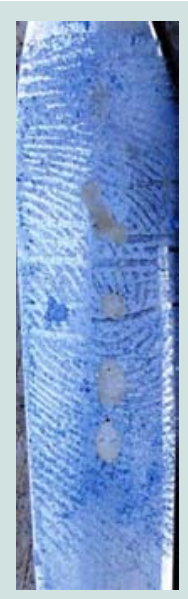

Figure 3: A sample fingerprint developed on blade of a knife after 15 days immersion in clean water. 
Citation: Sanjiv K, Sodhi GS, Kapoor S. A Multipurpose Composition Based on Brilliant Blue R for Developing Latent Fingerprints on Crime Scene Evidence. J Forensic Investigation. 2014;2(1): 3.

compared to electrical tape. Vehicle lifters often use electrical tapes to manipulate the digits on the number plates. After the vehicle is impounded, it is pertinent to develop fingerprints on the small strips of tape to identify the culprits. Figure 3 shows a representative fingerprint developed on a knife blade after immersion in clean water for 15 days. A sample fingerprint developed on, metallic surface and plastic tape after 5 days of immersion in dirty water respectively is shown in Figure 4. After a lapse of 15 days, the quality of developed fingerprints on tape becomes questionable. Figure 5 shows the impression on tape after 18 days of immersion in clean water.

The quality of developed fingerprints, both by the powder composition and by SPR formulation, was gauged on the basis of ridge clarity and degree of fluorescence, on a scale ranging from zero to 10 , according to the criteria laid down by SWGFAST [6]. The fingerprints developed within a period of 7 days by powder formulations, and by SPR formulation after 10 days of immersion in water may be accorded a scale of 7-10.

The raw materials used for preparing the present small

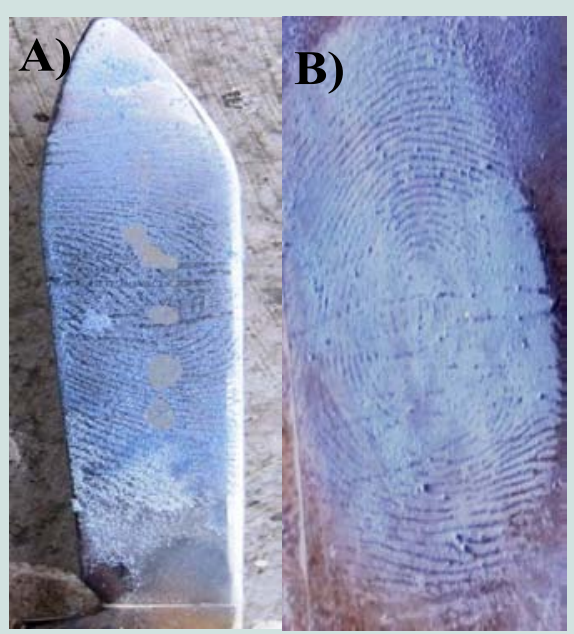

Figure 4: Fingerprints developed on $(A)$ metallic surface; $(B)$ plastic tape; after 5 days of immersion in dirty water.

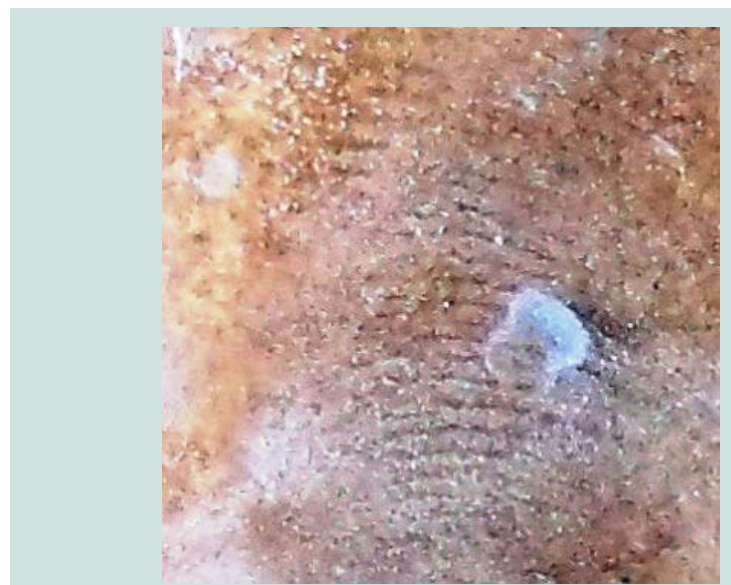

Figure 5: A fingerprint developed on tape after 18 days of immersion in clean water. compositions are cost-effective and easily available. These pose no occupational hazard to the user. Brilliant blue $\mathrm{R}$ is a non-toxic dye [7]. Zinc carbonate is an astringent and topical antiseptic. It does cause eye irritation [8]. Therefore, while using the powder formulation, the team members wore plain glasses. As a component of SPR formulation this was deemed unnecessary since a suspension of zinc carbonate in water would not injure the eyes. In comparison, molybdenum(IV) sulfide, which is used in conventional SPR formulation, is a skin irritant [9].

\section{Conclusion}

The multipurpose composition being reported in this communication, offers a convenient, cost-effective and efficient methodology to detect latent fingerprints on a broad spectrum of items. It may be used both as a powder as well as a small particle reagent. Its non-toxic nature notwithstanding, the composition has a relatively long shelf life of about 7 weeks as SPR suspension and for about one year as powder. Its ability to detect weak and faint fingerprints by virtue of its fluorescent characteristics not only enhances its utility, but also its potentiality in casework investigations.

\section{References}

1. RS Ramotowski (2013) in Lee and Gaensslen's Advances in Fingerprint Technology, (3 $3^{\text {rd }}$ edn), RS Ramotowski (Ed.), CRC Press, Boca Raton.

2. JP Mock (1984) Recent development on the use of small particle reagent Identification News 34: 2

3. A Frank, J Almog (1993) Modified SPR for latent fingerprint development on wet and dark items. J Forensic Identification 43: 240-244.

4. G Polimeni, FB Feudale, L Saravo, G De Fulvio (2004) A novel approach to identify the presence of fingerprints on wet surfaces. Forensic Sci Int 146: S45-46.

5. B Wilshire (1996) Advances in fingerprint detection. Endeavour 20:12-15.

6. (2013) SWGFAST, Standard for the Documentation of Analysis, Comparison, Evaluation, and Verification (ACE-V) (Latent), 2/12/10, version 1.0

7. FJ Green (1991) The Sigma-Aldrich Handbook of Stains, Dyes and Indicators, Aldrich Chemical Co., Inc., Milwaukee.

8. (2012) Safety Data Sheet: Zinc carbonate.

9. (2008) Material Safety Data Sheet: $\mathrm{BEMOL}_{\mathrm{TM}}$ Molybdenum Disulfide Powder. 